\title{
Bone resorption improvement by conditioned medium of stem cells from human exfoliated deciduous teeth in ovariectomized mice
}

\author{
AYAKO MAEDA $^{1}$, TAKASHI KIKUIRI ${ }^{1,2}$, YOSHITAKA YOSHIMURA ${ }^{3}$, \\ YASUTAKA YAWAKA $^{1}$ and TETSUO SHIRAKAWA ${ }^{2}$ \\ ${ }^{1}$ Department of Dentistry for Children and Disabled Individuals, Faculty of Dental Medicine and \\ Graduate School of Dental Medicine, Hokkaido University, Sapporo, Hokkaido 060-8586; \\ ${ }^{2}$ Department of Pediatric Dentistry, Nihon University School of Dentistry, Tokyo 101-8310; \\ ${ }^{3}$ Department of Pharmacology, Faculty of Dental Medicine and Graduate School of Dental Medicine, \\ Hokkaido University, Sapporo, Hokkaido 060-8586, Japan
}

Received November 1, 2021; Accepted January 14, 2022

DOI: $10.3892 / \mathrm{etm} .2022 .11228$

\begin{abstract}
Stem cells from human exfoliated deciduous teeth (SHED) are mesenchymal stem cells with multipotent differentiation potential present in the dental pulp tissue of the deciduous teeth. SHED produce secretions that have immunomodulatory and regenerative functions. In the present study, we investigated the effects of SHED-conditioned medium (SHED-CM) on osteopenia induced by the ovariectomy (OVX) phenotype and its corresponding immunological changes. Eleven-week-old female $\mathrm{C} 3 \mathrm{H} / \mathrm{HeJ}$ mice were subjected to OVX. SHED-CM was administered intraperitoneally in these mice for 4 weeks starting immediately after OVX. SHED-CM improved bone mass after OVX and elevated the polarization of M2 macrophages in the peritoneal cavity. SHED-CM also suppressed an OVX-induced increase in interferon- $\gamma(\mathrm{INF}-\gamma)$ and interleukin-17 (IL-17) concentrations in the peripheral blood. Inhibition of M2 macrophage polarization with neutralizing antibodies did not reduce the concentration of IFN- $\gamma$ and IL-17 in peripheral blood, which were increased by OVX, and did not alleviate osteopenia induced by the OVX phenotype. Mechanistically, these findings suggest that SHED-CM alleviates bone resorption by suppressing the activation of IFN- $\gamma$ and IL-17 cells by polarizing M2 macrophages. In conclusion, our data indicate that SHED-CM contains active secretions that may have promising efficacy to ameliorate OVX-induced osteopenia. We suggest that SHED-CM has the potential to be used as a novel therapeutic agent to inhibit osteoporosis.
\end{abstract}

Correspondence to: Dr Takashi Kikuiri, Department of Pediatric Dentistry, Nihon University School of Dentistry, 1-8-13 Kanda-surugadai, Chiyoda-ku, Tokyo 101-8310, Japan E-mail: kikuiri.takashi@nihon-u.ac.jp

Key words: stem cells from human exfoliated deciduous teeth, conditioned medium, osteoporosis, macrophage, INF- $\gamma$, IL-17

\section{Introduction}

Osteoporosis is a systemic skeletal disease characterized by low bone mass that increases bone fragility and the risk of fractures in patients (1). It increases due to estrogen deficiency after menopause, even though bone density in women is originally lower than in men (2). Various bone resorption inhibitors have been used to treat osteoporosis to prevent its progression (3). Because of the nature of the disease and the prolonged drug administration, osteoporosis drugs have a high bone resorption inhibitory effect and a high safety value for other tissues. The therapeutic agents frequently used in clinical practice, such as bisphosphonates and anti-receptor activator of nuclear factor- $\kappa \mathrm{B}$ ligand (RANKL) monoclonal antibodies, can reduce the risk of fragility fractures. However, they have marked side effects, such as inducing jaw osteonecrosis and increasing the risk of cancer and heart disease (4-6). Therefore, the development of safer and more effective therapeutic agents is an ongoing research effort.

Mesenchymal stem cells (MSCs) in the bone marrow and connective tissue reportedly exhibit immunosuppression in addition to pluripotency (7). For example, transplantation of bone marrow-derived MSCs in patients with autoimmune diseases, such as systemic lupus erythematosus and multiple sclerosis, has been shown to cause tolerance and alleviate symptoms in the recipients $(8,9)$. On the other hand, stem cells from human exfoliated deciduous teeth (SHED), which are present in the pulp tissue of deciduous teeth, are unique stem cells identified as a highly proliferative clonal cell population that can differentiate into a variety of cell types including neurons, adipocytes, osteoblasts, and endothelial cells (10). SHED are considered to be derived from the cranial neural crest and express early markers of both mesenchymal and neuroectodermal stem cells $(11,12)$. Furthermore, SHED have been reported to perform more potent immunomodulatory functions compared to bone marrow-derived MSCs (13). Currently, therapies that use the properties of MSCs, such as their pluripotency and immune control ability, are being tested 
for various diseases. For example, Yang et al (14) reported that SHED transplantation ameliorated glandular inflammation and dryness via soluble programmed cell death ligand 1 released from the transplanted SHED in mice exhibiting Sjögren's syndrome-induced dryness. Kitase et al (15) reported that SHED administration protected against cortical damage caused by hypoxic-ischemic encephalopathy and ameliorated behavioral deficits such as tetraplegia caused by cerebral ischemia in rats. Li et al (16) administered three intravenous doses of SHED over a six-week period in a cohort of 24 patients with type 2 diabetes on insulin therapy and followed these patients for 12 months. The authors reported that glycated serum albumin and glycated hemoglobin were normalized in 19 and 15 patients, respectively, following SHED transplantation.

Liu et al (17) reported that SHED transplantation improved the osteopenia phenotype in osteoporotic mice. The mechanism underlying suppressed osteoporosis included the induction of apoptosis of inflammatory by SHED transplantation via the Fas ligand (FasL)-mediated Fas pathway, with consequent suppression of osteoclast induction. The transplantation of SHED using their immunoregulatory ability is effective for the treatment of osteoporosis, although concerns remain regarding the safety of the transplanted cells, including tumor formation, which should be resolved before the implementation of SHED transplantation in clinical use. On the other hand, SEHD have been reported to release a variety of biologically active secreted factors, and studies in mice and rats have shown that the factors secreted from SHED are also highly effective in the treatment of various diseases such as neurodegenerative and autoimmune disorders, diabetes, and liver cirrhosis (18-21). These studies suggest that these factors secreted from SHED are effective for the treatment of many diseases. However, it remains unclear whether these factors secreted from SHED are effective in the treatment of osteoporosis. Therefore, we hypothesized that factors released from SHED would be effective in preventing osteoporosis. In the present study, we administered SHED-conditioned medium (CM) to ovariectomized (OVX) mice to determine its ability to prevent OVX-induced early osteoporosis phenotype.

\section{Materials and methods}

Cell culture. SHED were isolated and cultured from deciduous teeth (maxillary deciduous central incisors) donated by the Department of Pediatrics and Disabled Dentistry, Hokkaido University Hospital, under the approval of the Institutional Voluntary Clinical Research Review Board (approval no. 010-116). They were cultured with a minimum essential medium (a-MEM) (Invitrogen; Thermo Fisher Scientific, Inc.) supplemented with $20 \%$ fetal bovine serum (FBS) (Roche Diagnostics) in gas phase at $37^{\circ} \mathrm{C}$ with $5 \% \mathrm{CO}_{2}$ using a standardized protocol (10). Each cell was subcultured and replaced with serum-free a-MEM at $80 \%$ confluency, and culture supernatants were harvested after another $24 \mathrm{~h}$ of culture. The harvested culture supernatant was then centrifuged to remove cell debris.

Animal studies. The Hokkaido University Animal Experiment Committee approved this study (approval no. 15-0015). The animal experiments adhered to the Hokkaido University Animal Experiment Guidelines. We used 11-week-old female
C3H/HeJ mice (Sankyo Labo Service Corporation, Tokyo, Japan). We divided them into two groups: the OVX group, which underwent ovariectomy, and the Sham group, which underwent sham open surgery (17). Mice were anesthetized with sodium pentobarbital $(40 \mathrm{mg} / \mathrm{kg}$, intraperitoneal injection). The OVX procedures were performed to generate osteoporotic model mice. The OVX mice were further classified into three groups: OVX mice, the OVX serum-free medium (SF-MEM) mice that received $200 \mu 1$ of SF-MEM intraperitoneally, and the OVX SHED-CM mice that received $200 \mu 1$ of SHED-CM intraperitoneally. Both subgroups were administered a total of eight doses over 4 weeks, starting immediately after ovariectomy and proceeding twice weekly. At 4 weeks post-OVX, the mice were sacrificed using $\mathrm{CO}_{2}$ gas (flow rate was $30 \%$ displacement of the cage volume per min) for further examination.

MicroCT $(\mu C T)$ imaging. Femur and lumbar vertebrae underwent immersion fixation with $4 \%$ paraformaldehyde for 2 days after harvest. Fixed samples were subjected to $\mu \mathrm{CT}$ imaging using Latheta LCT200 (Hitachi, Japan) at a tube voltage of $50 \mathrm{kV}$ and a pixel size of $24.0 \mu \mathrm{m}$ according to a previously published protocol (18). Measurements in the photographed samples were taken using the analysis software included in the Latheta LCT200 system following the manufacturer's standards.

Histological analyses. After $\mu \mathrm{CT}$ imaging, femurs underwent demineralization with $10 \%$ ethylenediaminetetraacetic acid solution for 10 days, followed by alcoholic dehydration and paraffin embedding according to the flow cytometry method. Paraffin-embedded materials produced $5-\mu \mathrm{m}$ sections that were subjected to hematoxylin and eosin (H\&E) staining and tartrate-resistant acid phosphatase staining according to a conventional protocol (17). Image-Pro Premier (Media Cybernetics) was used for the uptake and analysis of section images. The lumbar spine was cut in the central region in the frontal transection orientation using a micro-cutting machine, BS-300CP (Meiwafosis, Japan), and the trabecular bone status was observed by stereomicroscopy.

Flow cytometry analysis. Peripheral blood was drawn from the fundus venous plexus using heparin-coated glass capillaries. The peripheral blood mononuclear cells (PBMNCs) were fixed and permeabilized using Cell Fixation and Permeabilization Kit (Abcam). Next, the PBMNCs were stained with PE/Cy7-labeled anti-IFN- $\gamma$ antibody (Biolegend) and PerCP/Cy5.5-labeled anti-IL-17A antibody (Biolegend). Peritoneal macrophages were collected via peritoneal lavage. Furthermore, $10 \mathrm{ml}$ of sterile phosphate-buffered saline was injected into the upper part of the abdominal cavity using a syringe. The abdomen of the mouse was massaged several times, and peritoneal lavage was performed. The cells were collected via centrifugation at $400 \mathrm{x}$ g for $10 \mathrm{~min}$ and then stained with PerCP/Cy5.5-labeled anti-CD80 (Biolegend) and APC-labeled anti-F4/80 antibody (Biolegend). Appropriate immunoglobulin (Ig) G-conjugated antibodies were used for isotype controls. Flow cytometry was performed on FACSVerse (BD Biosciences) and analyzed using FlowJo ver. 7.6 (BD Biosciences) using a standardized protocol (22). 
A

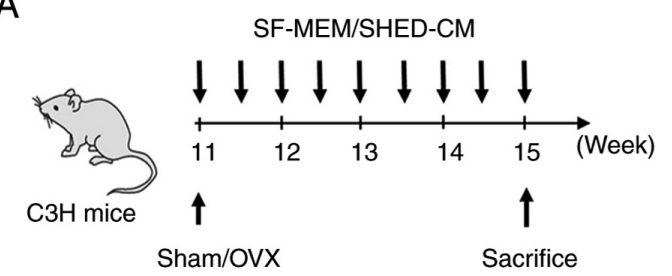

B

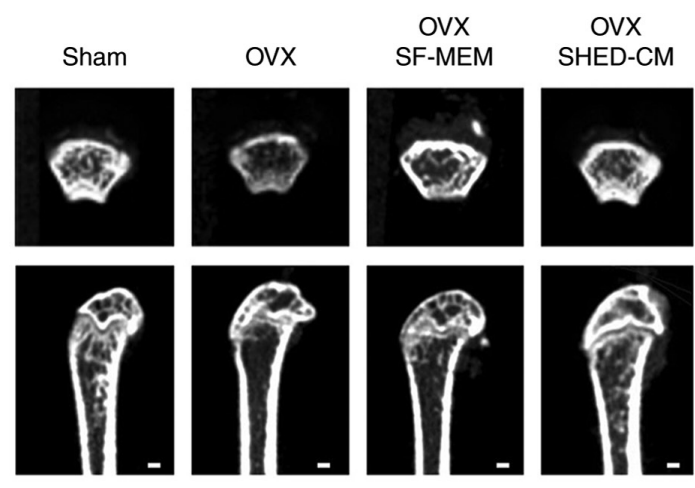

C

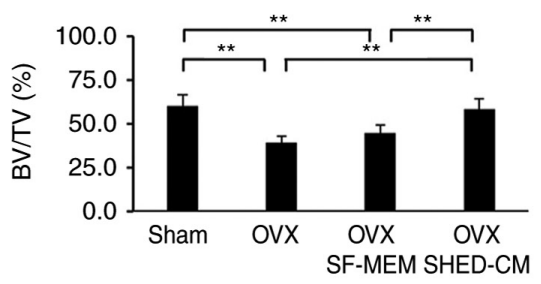

$\mathrm{D}$

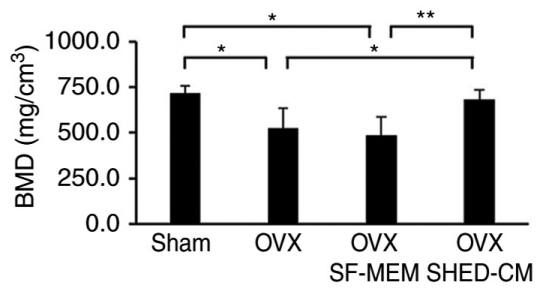

$\mathrm{E}$

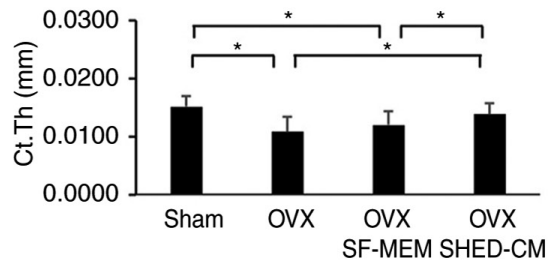

Figure 1. Experimental protocol and analysis of the femur with microCT $(\mu \mathrm{CT})$ imaging. SHED transplantation prevented the development of the osteoporotic phenotype in the OVX mice. (A) Schema indicating the experimental design for administering SHED-CM. (B) $\mu$ CT images of the cancellous bone architecture in the femur of the Sham, OVX, OVX SF-MEM and OVX SHED-CM mice. The cancellous bone volume in the OVX mice was observed to be decreased compared with that in the sham mice. The reduction in the cancellous bone volume induced by OVX in the OVX SHED-CM mice was partially restored. Scale bar, $100 \mu \mathrm{m}$. (C-E) $\mu \mathrm{CT}$ analysis revealed that BV/TV (C), BMD (D) and Ct.Th (E) after OVX were decreased. Administration of SHED-CM increased femoral BV/TV, BMD, and Ct.Th in the OVX mice. Data are expressed as mean $\pm \mathrm{SD}, \mathrm{n}=5$. ${ }^{*} \mathrm{P}<0.05,{ }^{* *} \mathrm{P}<0.01$. SHED, stem cells from human exfoliated deciduous teeth; OVX, ovariectomized; SF-MEM, serum-free medium; SHED-CM, SHED-conditioned medium; BV/TV, trabecular bone volume percentage; BMD, bone mineral density; Ct.Th, cortical thickness.

Enzyme-linked immunosorbent assay (ELISA). Interferon- $\gamma$ (IFN- $\gamma$ ), interleukin-17 (IL-17), and osteoprotegerin (OPG) levels in the blood serum samples were assayed using the Quantikine ELISA Kit (R\&D Systems) according to a standardized protocol (23).

Administration of IL-4-neutralizing antibody. For inhibiting the polarization of M2 macrophages, a neutralizing antibody (nAb) for IL-4 was injected. The OVX mice were intraperitoneally administered $25 \mu \mathrm{g}$ of either anti-mouse IL-4 antibody (U-CyTech Biosciences) or anti-rat $\operatorname{IgG}_{1}$ antibody as isotype control (U-CyTech) on the day after OVX. At 4 weeks post-OVX, the mice were sacrificed using $\mathrm{CO}_{2}$ gas for further examination.

Statistical analysis. The data are represented as mean \pm standard error of the mean. Statistical analyses of the results were performed using one-way analysis of variance (ANOVA) and post-hoc Tukey test and two-way repeated-measures ANOVA and post-hoc Bonferroni's test using the StatView ver. 5.0 (SAS Institute) software package. $\mathrm{P}<0.05$ was considered to indicate a statistically significant difference.

\section{Results}

Administration of SHED-CM prevents ovariectomy-induced bone loss. The present study investigated whether SHED-CM improved the osteoporotic phenotype by injecting OVX mice with SHED-CM and analyzing the treatment efficacy 4 weeks after OVX (Fig. 1A). $\mu \mathrm{CT}$ analysis revealed that the femur's trabecular bone volume fraction $(\mathrm{BV} / \mathrm{TV})$, bone mineral density (BMD), and average cortical thickness (Ct.Th) were increased in the OVX SHED-CM group compared with the OVX group (Fig. 1B-E). There was no significant difference between the OVX and the OVX SF-MEM groups. Histological analysis showed that the trabecular bone area in the OVX SHED-CM group was markedly elevated compared with that in the OVX group (Fig. 2A and B). Furthermore, the OVX SHED-CM group showed a significantly reduced number of tartrate-resistant acid phosphatase-positive cells in the distal femoral diaphysis compared with that in the OVX group (Fig. 2C and D). Observations on the lumbar spine's trabecular architecture showed that trabecular bone resorption in the central region was prominent in the OVX group (Fig. 2E and F). Despite performing OVX in the OVX SHED-CM group, their trabecular architecture was maintained in the same way as that in the Sham group.

SHED-CM inhibits osteopenia by suppressing T cell activation via M2 macrophages. The numbers of IFN- $\gamma$-producing cells and that of IL-17-producing cells in the peripheral blood were significantly increased in the OVX group and OVX SF-MEM group compared with the Sham group. The number of IFN- $\gamma$-producing cells and IL-17-producing cells in the OVX SHED-CM group were decreased compared with that in the OVX and the OVX SF-MEM group due to 
A
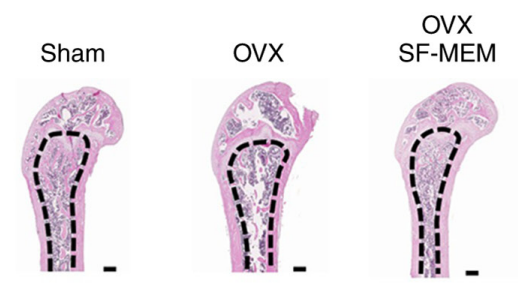

ovx

SHED-CM

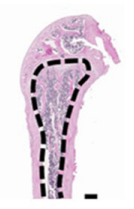

C
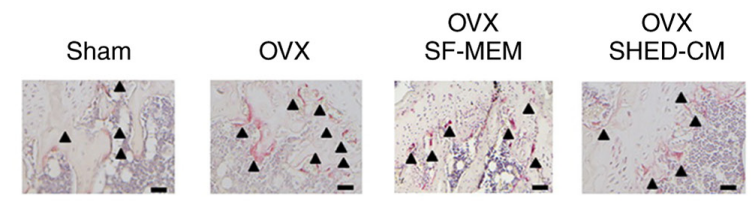

E

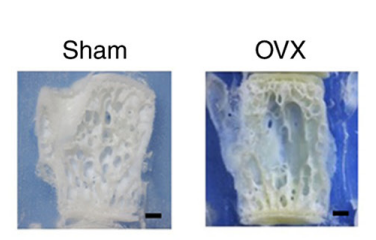

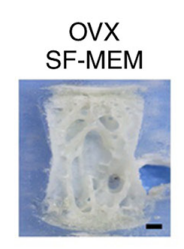

B

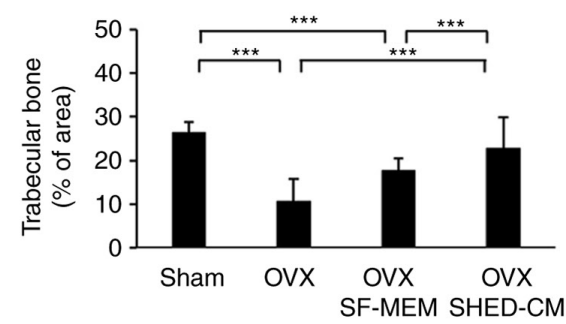

D

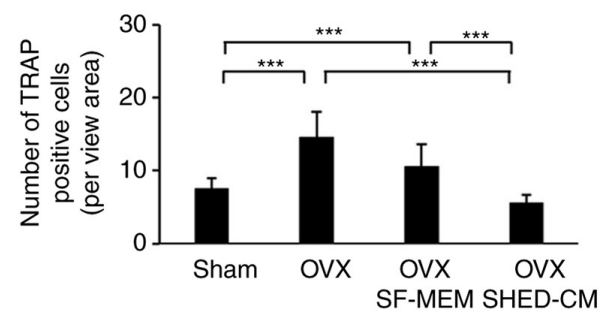

$\mathrm{F}$

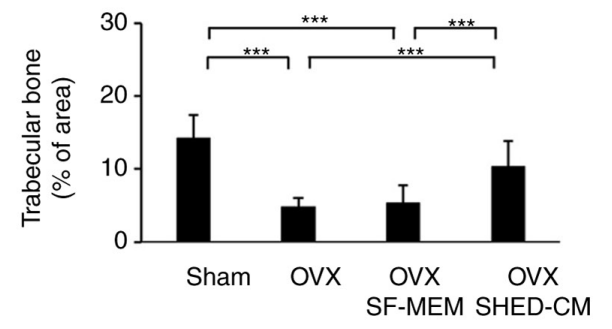

Figure 2. Histological analysis of the femur and lumbar spine. (A and B) Hematoxylin and eosin (H\&E) staining of the distal femur. The trabecular bone area (black broken lines) was reduced in the OVX mice compared with that noted in the sham mice. The administration of SHED-CM significantly increased the trabecular bone volume of the femur, which was significantly reduced in the OVX mice. (C and D) TRAP-positive cells (black arrows) was significantly increased in the femur of the OVX mice. Administration of SHED-CM reduced the number of TRAP positive cells in the femoral bone. (E and F) H\&E staining of the lumbar spine. OVX-induced resorption of the trabecular bone area in the lumbar spine was inhibited by the administration of SHED-CM. Scale bar, $100 \mu \mathrm{m}$. Data are expressed as mean $\pm \mathrm{SD}, \mathrm{n}=5$. ${ }^{* * *} \mathrm{P}<0.001$. OVX, ovariectomized; SF-MEM, serum-free medium; SHED-CM, conditioned medium of stem cells from human exfoliated deciduous teeth; TRAP, tartrate-resistant acid phosphatase.

the administration of SHED-CM (Fig. 3A-C). Furthermore, the analysis of the blood serum concentration of IFN- $\gamma$ and IL-17 using ELISA assay revealed that the IFN- $\gamma$ and IL-17 concentrations in the OVX group were significantly higher than those in the Sham group. Interestingly, the blood serum concentrations of IFN- $\gamma$ and IL-17 in the OVX SHED-CM group was markedly reduced compared with those in the OVX and the OVX SF-MEM group even though OVX was performed (Fig. 3D and E). Osteoprotegerin (OPG) concentrations in the blood serum of the OVX group mice and the OVX SHED-CM group were significantly decreased compared with those in the Sham group. Conversely, there was no significant difference between the OVX group and the OVX SHED-CM group in terms of OPG concentration (Fig. 3F). Regarding the distribution of macrophages in the peritoneal cavity, the expression rate of M1 macrophages $\left[\mathrm{F} 4 / 80^{+} \mathrm{CD} 80^{+}\right.$mononuclear cells (MNCs)] was not significantly different between the Sham $(49.4 \pm 4.3 \%)$ and the OVX $(70.3 \pm 4.9 \%)$ groups. In contrast, the expression rate was markedly decreased in the OVX SHED-CM $(40.5 \pm 10.5 \%)$ group compared with the $O V X(70.3 \pm 4.9 \%)$ and the SF-MEM (66.8 $\pm 8.3 \%$ ) groups (Fig. 3G and I). The expression rate of $\mathrm{M} 2$ macrophages $\left(\mathrm{F} 4 / 80^{+} \mathrm{CD} 206^{+} \mathrm{MNCs}\right)$ was not significantly different between the Sham $(38.9 \pm 4.8 \%)$ and the OVX (25.1 $\pm 3.8 \%)$ groups, whereas the expression rate was increased significantly in the OVX SHED-CM $(34.3 \pm 7.4 \%)$ compared with the OVX $(25.1 \pm 3.8 \%)$ and the OVX SF-MEM (25.8 5 5.1\%) (Fig. 3G-J).
Knockdown of M2 macrophages using IL-4 nAb. As per $\mu \mathrm{CT}$ images, the OVX anti-IL-4nAb mice as well as the OVX anti-IL-4 nAb SHED-CM mice showed considerable trabecular loss just below the growth plate (Fig. 4A and B). Analysis revealed that femur's trabecular bone volume fraction (BV/TV), bone mineral density (BMD), and average cortical thickness (Ct.Th) in the OVX IgG, the OVX IL-4 nAb and the OVX IL-4 nAb SHED-CM groups were significantly lower than those in the Sham group (Fig. 4C and D). BV/TV in the OVX IL-4 nAb, OVX IL-4 nAb SF-MEM and OVX IL-4 nAb SHED-CM groups was seen to significantly decrease compared that in the OVX IgG group (Fig. 4C). However, there was no significant difference in terms of BV/TV between the OVX IL-4 nAb, OVX IL-4 nAb SF-MEM and OVX IL-4 nAb SHED-CM groups (Fig. 4C). BMD was not significantly different between the OVX IgG, the OVX IL-4 nAb, and the OVX IL-4 nAb SHED-CM groups (Fig. 4D). Similarly, Ct.Th was not significantly different between the OVX IgG, the OVX IL-4 nAb SF-MEM, and the OVX IL-4 nAb SHED-CM groups (Fig. 4E).

Effect of IL-4 $n A b$ on the expression rate of M2 macrophages. The expression rate of M1 macrophage was significantly increased in the OVX IgG, the OVX IL-4 nAb, and the OVX IL-4 nAb SHED-CM groups compared with the Sham group (Fig. 5A).

The expression rate of $\mathrm{M} 1$ macrophages in the OVX IgG, the OVX IL-4 nAb, the OVX IL-4 nAb SF-MEM and the OVX IL-4 nAB SHED-CM were increased in the Sham 
A

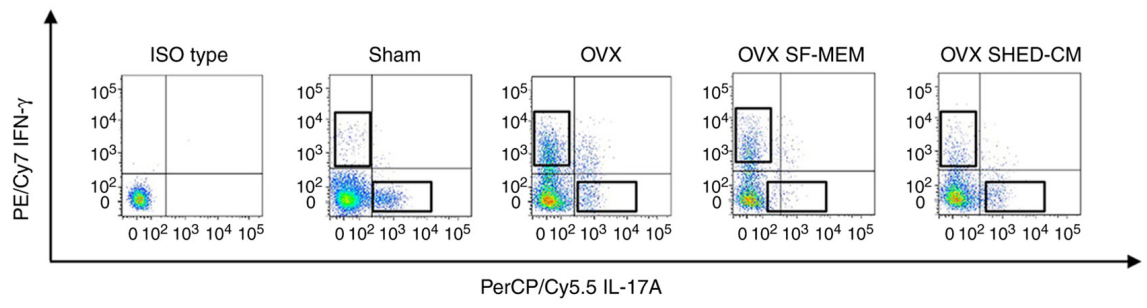

B

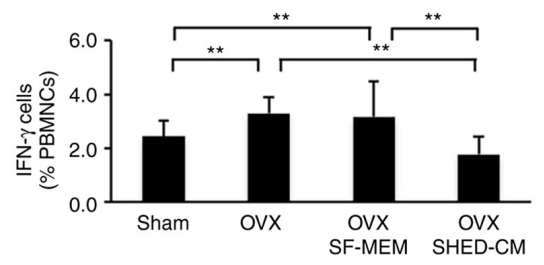

D

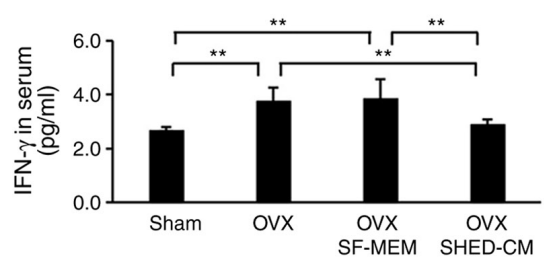

$\mathrm{F}$

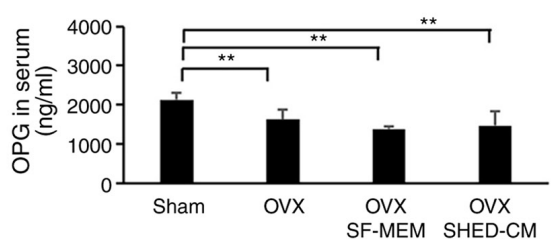

C

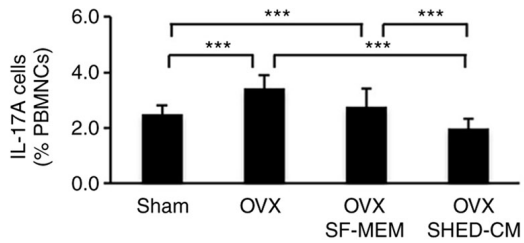

E

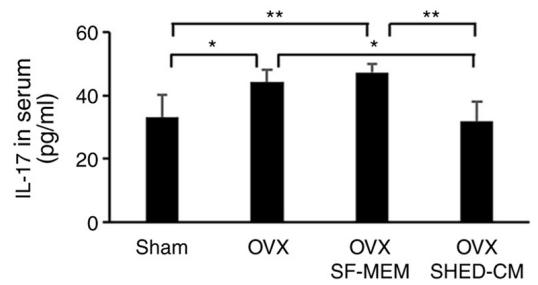

G

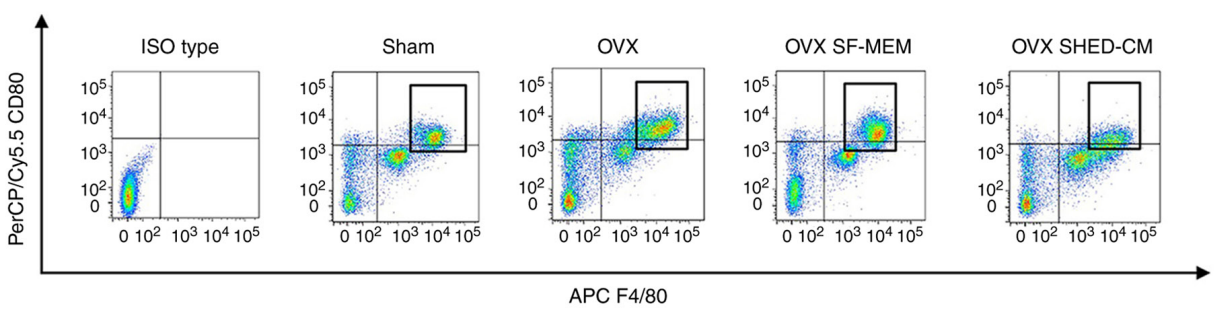

$\mathrm{H}$

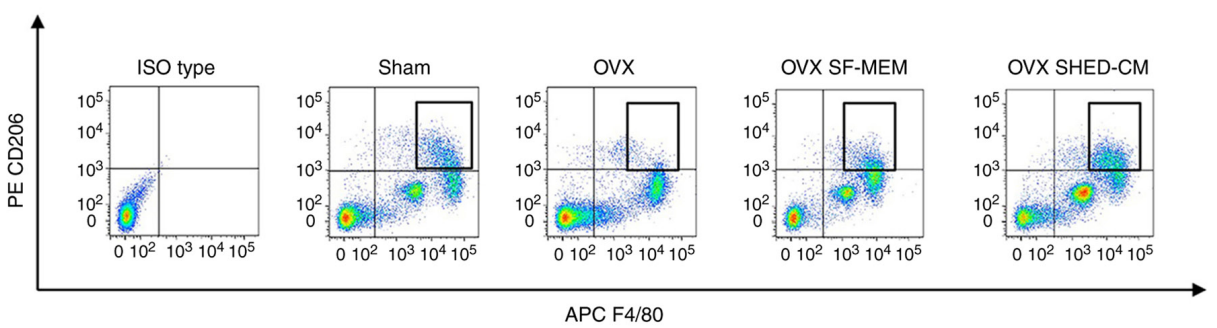

I

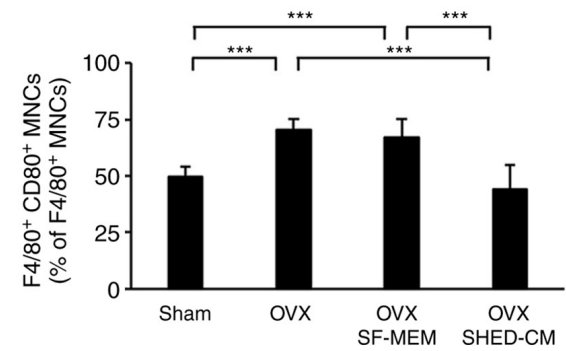

$\mathrm{J}$

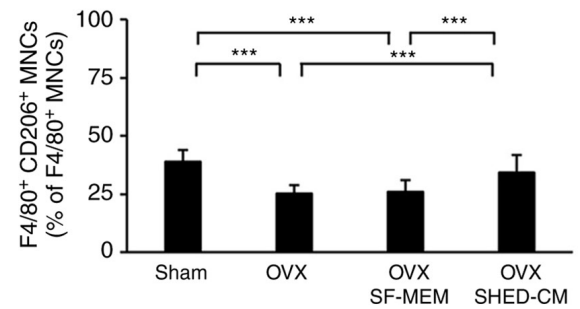

Figure 3. SHED-CM improved the balance between T cell subsets in the OVX mice and M1/M2 macrophages in the peritoneal cavity. (A-C) Flow cytometric analysis showed the percentages of IFN- $\gamma$ and IL-17 cells increased in the peripheral blood of the OVX mice. Administration of SHED-CM significantly decreased the percentages of IFN- $\gamma$ and IL-17 cells compared with OVX and the OVX SF-MEM mice. (D and E) ELSA showed increased concentrations of IFN- $\gamma$ and IL-17 in the serum of the OVX mice compared with those in the serum of the sham mice. After SHED-CM administration, the concentrations of IFN- $\gamma$ and IL-17 decreased markedly. (F) ELISA showed increased OPG concentrations in the serum of OVX mice compared with those in the serum of sham mice. After OVX, OPG concentrations decreased markedly. (G-J) Flow cytometric analysis showed that the percentage of M1 and M2 macrophages increased in the peritoneal cavity of the OVX mice. Administration of SHED-CM resulted in a reduction in the percentage of M1 macrophages. Data are expressed as mean $\pm \mathrm{SD}, \mathrm{n}=5 ;{ }^{*} \mathrm{P}<0.05,{ }^{* *} \mathrm{P}<0.01,{ }^{* * * *} \mathrm{P}<0.001$. SF-MEM, serum-free medium; SHED-CM, conditioned medium of stem cells from human exfoliated deciduous teeth; OVX, ovariectomized; PBMNCs, peripheral blood mononuclear cells; ELISA, enzyme-linked immunosorbent assay analysis; IFN- $\gamma$, interferon- $\gamma$; IL-17, interleukin-17; OPG, osteoprotegerin. 
A

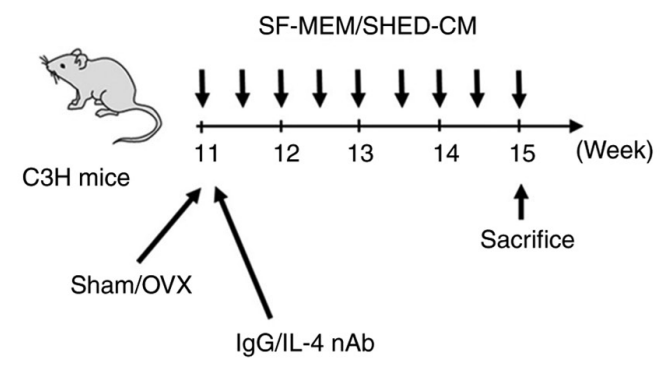

B

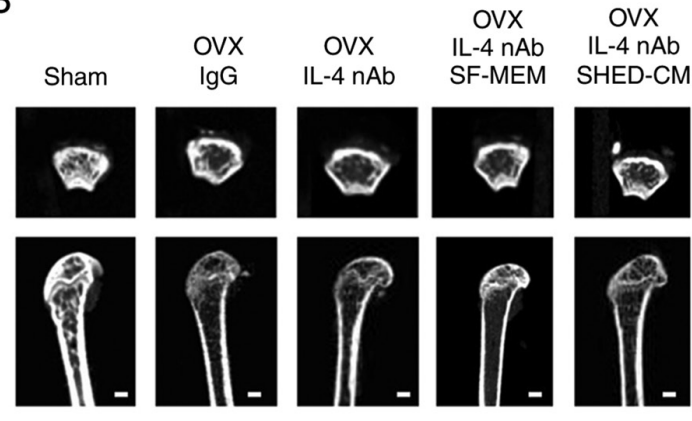

C

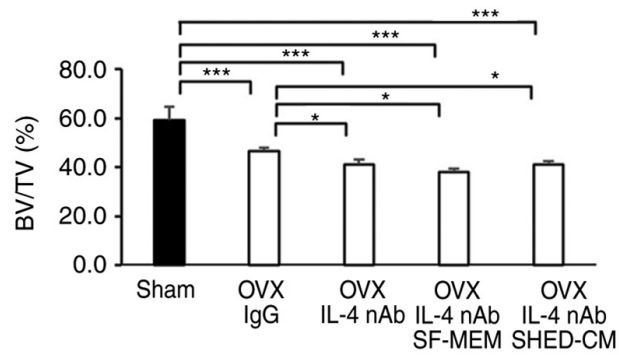

D

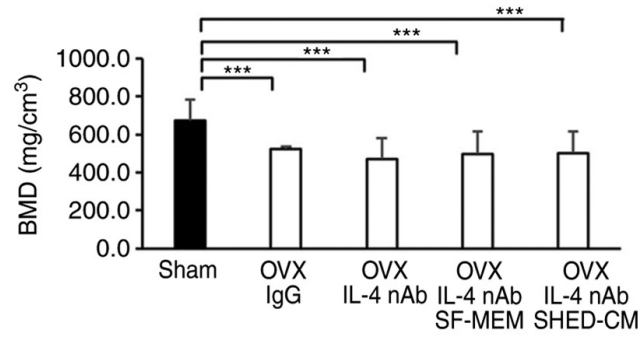

E

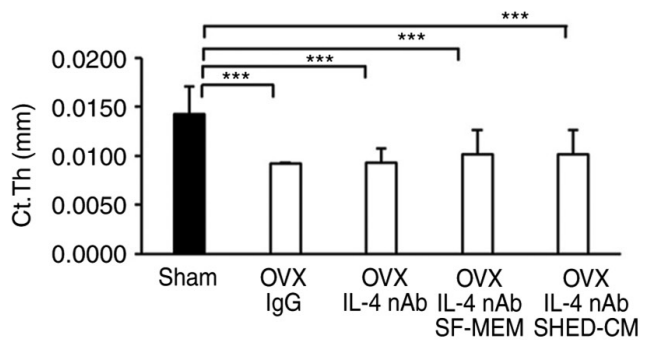

Figure 4. SHED-CM did not ameliorate the osteoporotic phenotype in the OVX mice with the knockdown of M2 macrophages using IL-4 nAb. (A) Schema indicating the experimental design for administering IL-4 nAb. (B) microCT $(\mu \mathrm{CT})$ imaging of cancellous bone architecture in the femur. (C-E) $\mu \mathrm{CT}$ analysis of BV/TV, BMD, and Ct.Th. Scale bar, $100 \mu \mathrm{m}$. Data are expressed as mean $\pm \mathrm{SD}, \mathrm{n}=5 ;{ }^{*} \mathrm{P}<0.05,{ }^{* * *} \mathrm{P}<0.001$. OVX, ovariectomized; IL-4 nAb, anti-interleukin-4-neutralizing antibody; IgG, anti-rat immunoglobulin $\mathrm{G}_{1}$ antibody; SF-MEM, serum-free medium; SHED-CM, conditioned medium of the stem cells from human exfoliated deciduous teeth; BV/TV, bone volume fraction; BMD, bone mineral density; Ct.Th, cortical thickness.

A
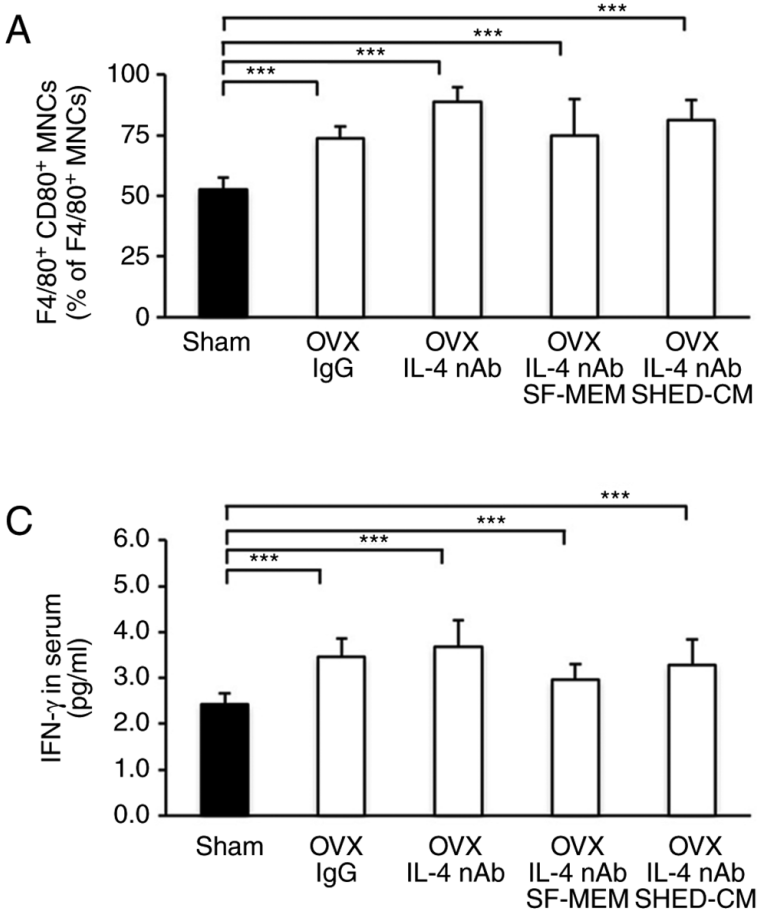

B
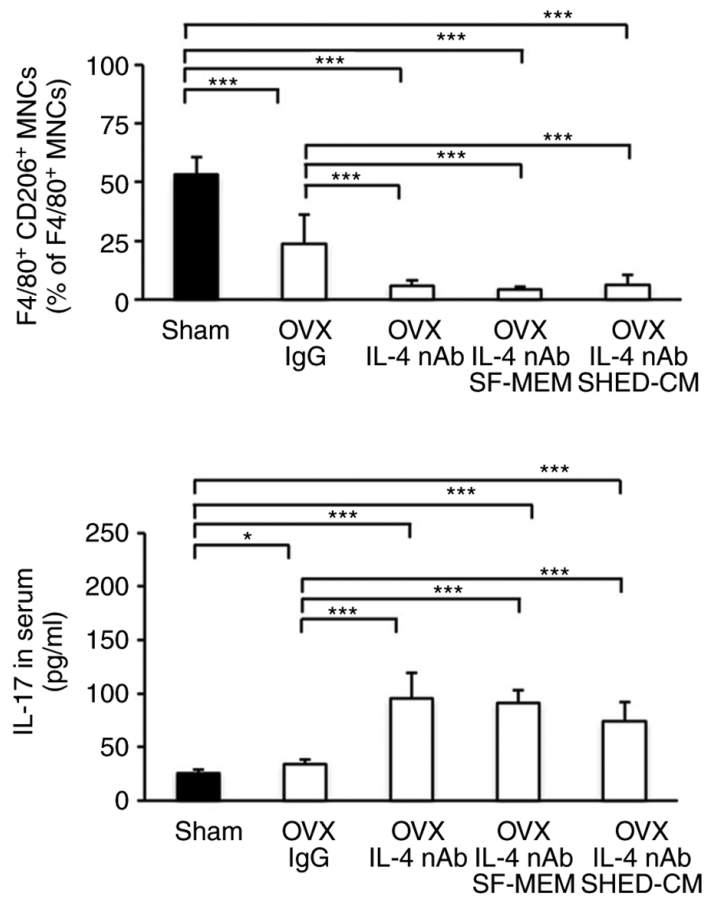

Figure 5. Effect of IL-4 nAb on the expression rate of M2 macrophages (F4/80+CD80- MNCs) and IFN- $\gamma$ and IL-17 serum concentrations. (A and B) The percentages of M1 and M2 macrophages according to flow cytometric analysis. (C and D) Concentrations of IFN- $\gamma$ and IL-17 in the serum. Data are expressed as mean $\pm \mathrm{SD}, \mathrm{n}=5 ;{ }^{*} \mathrm{P}<0.05,{ }^{* * * *} \mathrm{P}<0.001$. OVX, ovariectomized; IL-4-nAb, anti-interleukin-4-neutralizing antibody; IgG, anti-rat immunoglobulin $\mathrm{G}_{1} ; \mathrm{SF}-\mathrm{MEM}$, serum-free medium; SHED-CM, conditioned medium of the stem cells from human exfoliated deciduous teeth; PBMNCs, peripheral blood mononuclear cells; ELISA, enzyme-linked immunosorbent assay analysis; IFN- $\gamma$, interferon- $\gamma$; IL-17, interleukin-17. 
group (Fig. 5A). However, there was no significant difference in the expression rate of M1 macrophages between the OVX IL-4 nAb group and OVX IL-4 nAb SHED-CM group (Fig. 5A). Furthermore, the expression rate of M2 macrophages $\left(\mathrm{F} 4 / 80^{+} \mathrm{CD} 206^{+} \mathrm{MNCs}\right)$ was significantly decreased in the OVX IgG, the OVX IL-4 nAb, and the OVX IL-4 nAb SHED-CM groups compared with the Sham group (Fig. 5B). The expression rate of M2 macrophages in the OVX IL-4-nAb and the OVX IL-4 nAb SHED-CM groups were observed to significantly decrease compared with that in the OVX IgG group (Fig. 5B). However, there was no significant difference in the expression rate of $\mathrm{M} 2$ macrophages between the OVX IL-4 nAb and the OVX IL-4 nAb SHED-CM groups (Fig. 5B).

Effect of IL-4 nAb on IFN- $\gamma$ and IL-17 serum concentrations. The serum concentration of IFN- $\gamma$ in the OVX IgG, the OVX IL-4-nAb, and the OVX IL-4 nAb SHED-CM groups were significantly higher than that in the Sham group (Fig. 5C). However, the serum concentration of IFN- $\gamma$ was not significantly different between OVX IgG, the OVX IL-4 nAb, and the OVX IL-4 nAb SHED-CM groups (Fig. 5C). The serum concentration of IL-17 in the OVX IgG, the OVX IL-4-nAb, and the OVX IL-4 nAb SHED groups were significantly higher than that in the Sham group (Fig. 5D). Additionally, the serum concentration of IL-17 was significantly higher in the OVX IL-4 nAb and the OVX IL-4 nAb SHED-CM groups compared with the OVX IgG group (Fig. 5D).

\section{Discussion}

In the present study, human exfoliated deciduous teeth conditioned medium (SHED-CM) administration improved the early osteoporotic phenotype in ovariectomized (OVX) mice and exerted an inhibitory effect on bone resorption, in agreement with the findings of a previous study using systemic SHED injection (17). Animal models provide important insights in the study of the therapeutic modality of osteoporosis. Therefore, OVX mice have been considered the most important model for elucidating the cause of osteoporosis in humans and for the development of therapeutic agents (24). The cause of osteoporosis due to estrogen deficiency is not fully understood, but several mechanisms have been proposed to date. For example, estrogen deficiency causes chronic $\mathrm{T}$ cell activation and promotes bone resorption by osteoclasts $(25,26)$. In addition, murine studies have shown that increased concentrations of IFN-g and T helper 17 (Th17) produced by activated T cells are primarily associated with the development of osteoporosis (27-30). In the presence of estrogen deficiency, IFN- $\gamma$ promoted the expression of class II transactivator in immune cells to enhance antigen presentation between macrophages and $\mathrm{T}$ cells, which could upregulate tumor necrosis factor (TNF)- $\alpha$ and receptor activator for nuclear factor- $\kappa \mathrm{B}$ ligand (RANKL) and promote bone resorption (27). Furthermore, estrogen deficiency-induced increase in interferon- $\gamma$ (IFN- $\gamma$ ) has been demonstrated to promote bone loss by regulating osteoclastogenesis in mice (28). Tyagi et al (31) reported that estrogen deficiency led to increased differentiation of Th17 cells with related upregulation of signal transducer and activator of transcription 3 (STAT3), RAR-related orphan receptor (ROR)- $\gamma \mathrm{t}$, and ROR- $\alpha$ and induced bone loss by increasing the release of proosteoclastogenic cytokines including $\mathrm{TNF} \alpha$, IL-6, and RANKL from osteoblasts. Using flow cytometric analysis of T-cell subtypes in blood samples of women, Bhadricha et al (32) showed that Th17 cell frequency and IL-17 levels increased after menopause. In a previous study, SHED transplantation completely suppressed the osteopenia phenotype after OVX (17). The present study showed that cell-to-cell contacts between transplanted SHED and T cells activated FasL/Fas signaling and induced the apoptosis of T cells, which inhibited osteoclast differentiation by suppressing the increase in the plasma concentrations of IFN- $\gamma$ and IL-17. On the other hand, research has shown that inhibiting FasL/Fas signaling by SHED transplantation is very effective against osteoporosis as ovariectomy-induced osteopenia is not induced in Fas gene knockout mice (33). Interestingly, the mice treated with SHED in this study showed no progression of the osteopenia phenotype. SHED also suppressed the increase in plasma concentrations of IFN- $\gamma$ and IL-17 after ovariectomy. Thus, these data indicate that some secretions in the culture medium of SHED indirectly inhibited the increase in plasma concentrations of IFN- $\gamma$ and IL-17 after ovariectomy. These findings are consistent with the experimental findings of the previous study but suggest that a mechanism of action that is distinct from the FasL/Fas signaling pathway between transplanted SHED and T cells exists.

Although macrophages play a pivotal role in innate immunity, macrophages of various subtypes have been clearly shown to be involved in disease development and tissue healing (34). M1 macrophages activated during bacterial, viral, and allergic responses express several inflammatory cytokines and induce a phenotypic immune response $(35,36)$. M2 macrophages, which have anti-inflammatory and immunosuppressive functions, produce anti-inflammatory mediators to promote regression of the injury response and tissue repair (37-39). Therefore, we investigated how the administration of SHED-CM altered the expression ratio of M1 macrophages to M2 macrophages. M2 macrophages, which were only scarcely present in the sham and OVX mice, were significantly increased in the SHED-CM mice. In addition, to clarify whether M2 macrophages were involved in improving the osteopenia phenotype induced by OVX, we administered the $\mathrm{nAb}$ of IL-4 required for the polarization of M2 macrophages and evaluated the expression of M2 macrophages. In the OVX anti-IL-4 nAb SHED-CM mice, the osteopenia phenotype induced by ovariectomy was not improved, even though SHED-CM was administered.

The polarization of M2 macrophages was suppressed in the mice treated with a neutralizing antibody (nAb), as their ratio of M2 macrophages to M1 macrophages decreased. In addition, the serum concentration of IFN- $\gamma$ and that of Th17 were increased significantly in the mice treated with IL-4 nAb compared with the sham mice. The increases in IFN- $\gamma$ and Th17 may be due to the inhibition of M2 macrophage polarization by the effects of the $\mathrm{nAb}$.

In the present study, we did not identify the active factor that mitigates bone resorption in SHED-CM. However, Hiraki et al (40) reported that SHED-CM contains bone metabolism-related factors, angiogenesis-related factors, and neurotrophic factors. Moreover, MSC-CM contains insulin-like growth factor-1, transforming growth factor (TGF)- $\beta 1$, and vascular endothelial growth factor (VEGF), and these factors 
affect the regeneration of bone cells (41). It is possible that these cytokines in SHED-CM may have acted to improve osteoporosis in this experiment. In addition, several other factors may have cooperated to activate various signaling pathways; those affecting the improvement of osteoporosis need further investigation.

We determined the therapeutic efficacy of SHED-CM on osteoporosis caused by estrogen deficiency in OVX mice. Our results showed that SHED-CM inhibited osteoclast differentiation by suppressing IFN- $\gamma$ and IL-17 cells by inducing M2 macrophage polarization, thereby improving the pathology of OVX-induced osteoporosis. In conclusion, we suggest that SHED-CM has the potential to be used as a therapeutic agent targeting the inhibition of bone resorption, which is an important therapeutic strategy for osteoporosis.

\section{Acknowledgements}

Not applicable.

\section{Funding}

This study was supported in part by KAKENHI (Grant-in-Aid for Scientific Research from the Japan Society for the Promotion of Science: 20K10219, 19K10280, 21K10172).

\section{Availability of data and materials}

The datasets used and/or analyzed during the current study are available from the corresponding author on reasonable request.

\section{Authors' contributions}

AM, TK, and YYo performed the animal experiments, histological analyses and flow cytometry analysis. TK and YYo conducted the statistical analysis. YYa and TS performed the $\mu \mathrm{CT}$ study. AM and TK wrote the manuscript. AM, TK, and YYa conceived the study and participated in its design and coordination. All authors confirm the authenticity of all the raw data. All authors read and approved the final manuscript for publication.

\section{Ethics approval and consent to participate}

SHED were isolated and cultured from deciduous teeth donated by the Department of Pediatrics and Disabled Dentistry, Hokkaido University Hospital (Kita-ku, Sapporo, Japan) under the approval of the Institutional Voluntary Clinical Research Review Board (approval no. 010-116). The study protocols for the animal experiments were approved by The Hokkaido University Animal Experiment Committee. All animal experiments adhered to Hokkaido University Animal Experiment Guidelines.

\section{Patient consent for publication}

Not applicable.

\section{Competing interests}

The authors declare that they have no competing interest.

\section{References}

1. Kanis JA, McCloskey EV, Harvey NC, Johansson H and Leslie WD: Intervention thresholds and the diagnosis of osteoporosis. J Bone Miner Res 30: 1747-1753, 2015.

2. Khosla S, Atkinson EJ, Melton LJ III and Riggs BL: Effects of age and estrogen status on serum parathyroid hormone levels and biochemical markers of bone turnover in women: A population-based study. J Clin Endocrinol Metab 82: 1522-1527, 1997.

3. Vidal M, Thibodaux RJ, Neira LFV and Messina OD: Osteoporosis: A clinical and pharmacological update. Clin Rheumatol 38: 385-395, 2019.

4. Marx RE: Pamidronate (Aredia) and zoledronate (Zometa) induced avascular necrosis of the jaws: A growing epidemic. J Oral Maxillofac Surg 61: 1115-1117, 2003.

5. McClung M, Harris ST, Miller PD, Bauer DC, Davison KS, Dian L, Hanley DA, Kendler DL, Yuen CK and Lewiecki EM: Bisphosphonate therapy for osteoporosis: Benefits, risks, and drug holiday. Am J Med 126: 13-20, 2013.

6. Deligiorgi MV and Trafalis DT: The safety profile of denosumab in oncology beyond the safety of denosumab as an anti-osteoporotic agent: Still more to learn. Expert Opin Drug Saf 20: 191-213, 2021.

7. Akiyama K, Chen C, Wang D, Xu X, Qu C, Yamaza T, Cai T, Chen W, Sun L and Shi S: Mesenchymal-stem-cell-induced immunoregulation involves FAS-ligand-/FAS-mediated T cell apoptosis. Cell Stem Cell 10: 544-555, 2012.

8. Tang WY, Liu JH, Peng CJ, Liao Y, Luo JS, Sun X, Tang YL and Luo XQ: Functional characteristics and application of mesenchymal stem cells in systemic lupus erythematosus. Arch Immunol Ther Exp (Warsz) 69: 7, 2021.

9. Barati S, Tahmasebi F and Faghihi F: Effects of mesenchymal stem cells transplantation on multiple sclerosis patients. Neuropeptides 84: 102095, 2020.

10. Miura M, Gronthos S, Zhao M, Lu B, Fisher LW, Robey PG and Shi S: SHED: Stem cells from human exfoliated deciduous teeth. Proc Natl Acad Sci USA 100: 5807-5812, 2003.

11. Sakai VT, Zhang Z, Dong Z, Neiva KG, Machado MA, Shi S, Santos CF and Nör JE: SHED differentiate into functional odontoblasts and endothelium. J Dent Res 89: 791-796, 2010.

12. Chadipiralla K, Yochim JM, Bahuleyan B, Huang CY, Garcia-Godoy F, Murray PE and Stelnicki EJ: Osteogenic differentiation of stem cells derived from human periodontal ligaments and pulp of human exfoliated deciduous teeth. Cell Tissue Res 340: 323-333, 2010.

13. Yamaza T, Kentaro A, Chen C, Liu Y, Shi Y, Gronthos S, Wong S and Shi S: Immunomodulatory properties of stem cells from human exfoliated deciduous teeth. Stem Cell Res Ther 1: 5, 2010.

14. Yang N, Liu X, Chen X, Yu S, Yang W and Liu Y: Stem cells from exfoliated deciduous teeth transplantation ameliorates Sjögren's syndrome by secreting soluble PD-L1. J Leukoc Biol: Oct 8, 2021 (Epub ahead of print).

15. Kitase Y, Sato Y, Ueda K, Suzuki T, Mikrogeorgiou A, Sugiyama Y, Matsubara K, Tsukagoshi Okabe Y, Shimizu S, Hirata $\mathrm{H}$, et al: A novel treatment with stem cells from human exfoliated deciduous teeth for hypoxic-ischemic encephalopathy in neonatal rats. Stem Cells Dev 29: 63-74, 2020.

16. Li W, Jiao X, Song J, Sui B, Guo Z, Zhao Y, Li J, Shi S and Huang Q: Therapeutic potential of stem cells from human exfoliated deciduous teeth infusion into patients with type 2 diabetes depends on basal lipid levels and islet function. Stem Cells Transl Med 10: 956-967, 2021.

17. Liu Y, Wang L, Liu S, Liu D, Chen C, Xu X, Chen X and Shi S: Transplantation of SHED prevents bone loss in the early phase of ovariectomy-induced osteoporosis. J Dent Res 93: 1124-1132, 2014.

18. Mita T, Furukawa-Hibi Y, Takeuchi H, Hattori H, Yamada K, Hibi $\mathrm{H}$, Ueda $\mathrm{M}$ and Yamamoto A: Conditioned medium from the stem cells of human dental pulp improves cognitive function in a mouse model of Alzheimer's disease. Behav Brain Res 293: 189-197, 2015.

19. Bartholomew A, Sturgeon C, Siatskas M, Ferrer K, McIntosyu K, Patil S, Hardy W, Devine S, Ucker D, Deans R, et al: Mesenchymal stem cells suppress lymphocyte proliferation in vitro and prolong skin graft survival in vivo. Exp Hematol 30: 42-48, 2002.

20. He Q, Wang L, Zhao R, Yan F, Sha S, Cui C, Song J, Hu H, Guo X, Yang M, et al: Mesenchymal stem cell-derived exosomes exert ameliorative effects in type 2 diabetes by improving hepatic glucose and lipid metabolism via enhancing autophagy. Stem Cell Res Ther 11: 223, 2020. 
21. Li T, Yan Y, Wang B, Qian H, Zhang X, Shen L, Wang M, Zhou Y, Zhu W, Li W and Xu W: Exosomes derived from human umbilical cord mesenchymal stem cells alleviate liver fibrosis. Stem Cells Dev 22: 845-854, 2013.

22. Lubura M, Hesse D, Neumann N, Scherneck S, Wiedmer P and Schürmann A: Non-invasive quantification of white and brown adipose tissues and liver fat content by computed tomography in mice. PLoS One 7: e37026, 2012.

23. Fathi E, Farahzadi R and Valipour B: Alginate/gelatin encapsulation promotes NK cells differentiation potential of bone marrow resident C-kit ${ }^{+}$hematopoietic stem cells. Int J Biol Macromol 177: 317-327, 2021.

24. Komori T: Animal models for osteoporosis. Eur J Pharmacol 759: 287-294, 2015

25. Fathi E, Farahzadi R, Vietor I and Javanmardi S: Cardiac differentiation of bone-marrow-resident c-kit ${ }^{+}$stem cells by L-carnitine increases through secretion of VEGF, IL6, IGF-1, and TGF- $\beta$ as clinical agents in cardiac regeneration. J Biosci 45: 92, 2020

26. Pacifici R: Estrogen deficiency, T cells and bone loss. Cell Immunol 252: 68-80, 2008.

27. Fischer V and Haffner-Luntzer M: Interaction between bone and immune cells: Implications for postmenopausal osteoporosis. Semin Cell Dev Biol: May 20, 2021 (Epub ahead of print).

28. Cenci S, Toraldo G, Weitzmann MN, Roggia C, Gao Y, Qian WP, Sierra O and Pacifici R: Estrogen deficiency induces bone loss by increasing $\mathrm{T}$ cell proliferation and lifespan through IFN-gamma-induced class II transactivator. Proc Natl Acad Sci USA 100: 10405-10410, 2003

29. Gao Y, Grassi F, Ryan MR, Terauchi M, Page K, Yang X, Weitzmann MN and Pacifici R: IFN-gamma stimulates osteoclast formation and bone loss in vivo via antigen-driven $\mathrm{T}$ cell activation. J Clin Invest 117: 122-132, 2007.

30. Zhao R: Immune regulation of bone loss by Th17 cells in oestrogen-deficient osteoporosis. Eur J Clin Invest 43: 1195-1202, 2013.

31. Tyagi AM, Srivastava K, Mansoori MN, Trivedi R, Chattopadhyay $\mathrm{N}$ and Singh D: Estrogen deficiency induces the differentiation of IL-17 secreting Th17 cells: A new candidate in the pathogenesis of osteoporosis. PLoS One 7: e44552, 2012.
32. Bhadricha H, Patel V, Singh AK, Savardekar L, Patil A, Surve S and Desai M: Increased frequency of Th17 cells and IL-17 levels are associated with low bone mineral density in postmenopausal women. Sci Rep 11: 16155, 2021.

33. Kovacic N, Grcevic D, Katavic V, Lukic IK, Grubisic V, Mihovilovic K, Cvija H, Croucher PI and Marusic A: Fas receptor is required for estrogen deficiency-induced bone loss in mice. Lab Invest 90: 402-413, 2010.

34. Murray PJ and Wynn TA: Protective and pathogenic function of macrophage subsets. Nat Rev Immunol 11: 723-737, 2011.

35. Lu LY, Loi F, Nathan K, Lin TH, Pajarinen J, Gibon E, Nabeshima A, Cordova L, Jämsen E, Yao Z and Goodman SB: Pro-inflammatory M1 macrophages promote Osteogenesis by mesenchymal stem cells via the COX-2-prostaglandin E2 pathway. J Orthop Res 35: 2378-2385, 2017.

36. Sica A and Mantovani A: Macrophage plasticity and polarization: In vivo veritas. J Clin Invest 122: 787-795, 2012.

37. Liu Q, Tian Y, Zhao X, Jing H, Xie Q, Li P, Li D, Yan D and Zhu X: NMAAP1 expressed in BCG-activated macrophage promotes M1 macrophage polarization. Mol Cells 38: 886-894, 2015.

38. van Dalen FJ, van Stevendaal MHME, Fennemann FL, Verdoes M and Ilina O: Molecular repolarisation of tumour-associated macrophages. Molecules 24: 9, 2018.

39. Mills CD: M1 and M2 macrophages: Oracles of health and disease. Crit Rev Immunol 32: 463-488, 2012.

40. Hiraki T, Kunimatsu R, Nakajima K, Abe T, Yamada S, Rikitake $\mathrm{K}$ and Tanimoto K: Stem cell-derived conditioned media from human exfoliated deciduous teeth promote bone regeneration. Oral Dis 26: 381-390, 2020.

41. Ando Y, Matsubara K, Ishikawa J, Fujio M, Shohara R, Hibi H, Ueda $\mathrm{M}$ and Yamamoto A: Stem cell-conditioned medium accelerates distraction osteogenesis through multiple regenerative mechanisms. Bone 61: 82-90, 2014.

This work is licensed under a Creative Commons Attribution-NonCommercial-NoDerivatives 4.0 International (CC BY-NC-ND 4.0) License. 